\title{
Update on biomarkers for amyloid pathology in Alzheimer's disease
}

Nicholas J. Ashton 1,2,3,4*, Michael Schöll ${ }^{3,5}$, Kerstin Heurling ${ }^{3}$, Eleni Gkanatsiou ${ }^{4}$, Erik Portelius ${ }^{4,6}$, Kina Höglund ${ }^{4}, 6$, Gunnar Brinkmalm ${ }^{4,6}$, Abdul Hye ${ }^{1,2}$, Kaj Blennow ${ }^{4,6}$ and Henrik Zetterberg ${ }^{4,6,7,8}$

${ }^{1}$ Old Age Psychiatry, Institute of Psychiatry, Psychology and Neuroscience, Maurice Wohl Institute Clinical Neuroscience Institute, King's College London, London, UK

${ }^{2}$ NIHR Biomedical Research Centre for Mental Health and Biomedical Research Unit for Dementia at South London and Maudsley NHS Foundation, London, UK

${ }^{3}$ Wallenberg Centre for Molecular and Translational Medicine and the Department of Psychiatry and Neurochemistry, University of Gothenburg, Gothenburg, Sweden

${ }^{4}$ Department of Psychiatry and Neurochemstry, Institute of Neuroscience and Physiology, the Sahlgrenska Academy at the University of Gothenburg, Mölndal, Sweden

${ }^{5}$ Clinical Memory Research Unit, Department of Clinical Sciences, Malmö, Lund University, Lund, Sweden

${ }^{6}$ Clinical Neurochemistry Laboratory, Sahlgrenska University Hospital, Mölndal, Sweden

${ }^{7}$ Department of Molecular Neuroscience, UCL Institute of Neurology, Queen Square, London, UK

${ }^{8}$ UK Dementia Research Institute at UCL, London, UK

*Corresponding author: Nicholas J. Ashton (nicholas.ashton@gu.se)

Word Count: 5,123 


\begin{abstract}
At the centre of Alzheimer's disease (AD) pathogenesis is the aberrant aggregation of amyloid$\beta(\mathrm{A} \beta)$ into oligomers, fibrils, and plaques. Effective monitoring of $\mathrm{A} \beta$ deposition directly in patients is essential to assist anti-A $\beta$ therapeutics in target engagement and participant selection. In the advent of approved anti-A $\beta$ therapeutics, biomarkers will become of fundamental importance in initiating treatments having disease modifying effects at the earliest stage. Two well established $A \beta$ biomarkers are widely utilised: $A \beta$-binding ligands for positron emission tomography (PET) and immunoassays to measure $\mathrm{A} \beta 42$ in cerebrospinal fluid (CSF). In this review, we will discuss the current clinical, diagnostic, and research state of biomarkers for $A \beta$ pathology. Furthermore, we will explore the current application of blood-based markers to assess $A \beta$ pathology.
\end{abstract}

Key Words: amyloid- $\beta$ (A $\beta)$, Alzheimer's disease, dementia, biomarkers, cerebrospinal fluid, positron emission tomography, blood-based biomarkers 


\section{Introduction}

Alzheimer's disease (AD) is the predominant cause of dementia, characterised by memory impairment and cognitive decline which severely impacts on daily living. AD is an age-related, progressive and irreversible neurodegenerative disorder that eventually leads to synaptic dysfunction and neuronal damage in regions of the brain essential for higher cognitive function. The socioeconomic consequence of $\mathrm{AD}$ is of great concern. There are more than 40 million worldwide dementia sufferers, mostly older than 60 years of age, a number which is expected to exceed 115 million by 2050 [1]. Alarmingly, these projected increases in dementia prevalence are proportionally much higher for developing countries than for Western Europe and the USA, which already have much older populations [2]. The global economic burden of dementia care is currently estimated at $\$ 604$ billion and in a similar fashion to prevalence rates this figure is set to rapidly increase, with a projected $85 \%$ rise in costs within the next 20 years.

Still to this date the diagnosis of AD can only be confirmed, with any certainty, by histological examination of brain tissue at autopsy. In addition to gross cortical atrophy, widespread cellular degeneration and neuronal loss, pathological inspection should demonstrate substantial evidence of the classical pathological hallmarks of $\mathrm{AD}$; intracellular neurofibrillary tangles (NFT) consisting of hyperphosphorylated tau protein and extracellular amyloid plaques. Plaques consist of extracellular aggregates of the 42 amino acid-long amyloid- $\beta$ peptide (A $\beta 42$ ), as well as $\mathrm{N}$-terminally truncated forms thereof, a consequence of proteolytic cleavage of amyloid precursor protein (APP) [3]. Thus far, genetic (reviewed in [4]), biochemical (reviewed in [5]), pathological (reviewed in [6]) and cellular (reviewed in [7]) studies strongly indicate that the disparity in the production and effective clearance of $A \beta$ is the fundamental event that leads to dementia $[8,9]$. Alternative hypotheses suggest that $A \beta$ is a necessary but not sufficient event leading to dementia and its accumulation is in parallel with other pathological events $[2,10,11]$. As reviewed below in greater detail, some researchers describe diffusible $\mathrm{A} \beta$ aggregates as causative agents in a linear model of $\mathrm{AD}$ pathogenesis [5], whereas others argue for a more complex model in which $A \beta$ deposition and racemization are a match that eventually induce critical downstream changes, including tau phosphorylation, microglial activation and cerebrovascular changes, that together interact to cause neurodegeneration [7]. According to this model, the direction of causality may vary, at least in the sporadic form of the disease; for example, cerebrovascular changes may induce amyloidogenic APP-processing and $A \beta$ aggregation, opening the possibility for vicious feed-forward loops. 
Although an age-related disease, usually affecting people over the age of 65 , it has been widely shown that the accumulation of $A \beta$ plaques begins 15-20 years prior to clinical presentation [12] and have reached a plateau when the onset of cognitive, functional and behavioural decline occurs [13] making an early diagnosis problematic. The clinical diagnosis for probable AD is still largely based upon progressive memory impairment and a decline in cognitive state. Recent modifications to the diagnostic criteria have recognised the significant role of biomarkers assessing $\mathrm{A} \beta$ by in vivo positron emission tomography (PET) and cerebrospinal fluid (CSF) analysis for preclinical, prodromal and phenotypical AD [14] . In the same manner, $\mathrm{A} \beta$ imaging and fluid biomarkers are fundamental inclusion criterion to support diseasespecific trials, targeting the amyloid hypothesis, in participant selection and proof of target engagement [15]. In this review, we will discuss the current state of biomarkers for $\mathrm{A} \beta$ pathology, including their diagnostic performance and how they can be used in clinical diagnostic and research settings to support or exclude a diagnosis of AD.

\section{A $\beta$ in Alzheimer's disease}

Research advances during the past two decades have produced detailed knowledge on the underlying disease mechanism of $\mathrm{AD}$, which is centred around the proteolytic cleavage of APP. Typically, the cleavage of APP occurs by the sequential action of $\alpha$-secretase and $\gamma$-secretase to produce non-amyloidogenic products (the non-amyloidogenic pathway). The initial cleavage of $\alpha$-secretase releases a large soluble ectodomain of APP ( $\operatorname{APP} \alpha)$ and a carboxyl terminal fragment $(\alpha \mathrm{CTF})[16]$ which is further cleaved by $\gamma$-secretase to produce the APP intracellular cytoplasmic domain (AICD) and soluble peptide P3 [17]. However, a proportion of APP is cleaved by $\beta$-secretase (BACE1) to produce sAPP $\beta$ and $\beta C T F$. The subsequent cleavage by $\gamma$ secretase produces $\mathrm{A} \beta$ fragments and AICD which are transported, via exocytosis, to the extracellular space $[16,18,19]$. The predominant forms of $A \beta$ produced by the amyloidogenic pathway are $A \beta 38, A \beta 40$ and $A \beta 42$, of which the latter has a tendency to aggregate, working as a seed for plaque deposition [20] but also $A \beta$-approximate peptides exist as neurotoxic dimers, oligomers, protofibrils and fibrils [21, 22]. Subsequent diffuse A $\beta$ plaque formation leads to localised microglial activation, cytokine release, astrocytosis and a multi-protein inflammatory response $[23,24]$. The "amyloid cascade hypothesis", has been the prevailing theory to explain $\mathrm{AD}$ pathogenesis [9]. This model suggests that $\mathrm{A} \beta$ aggregation acts as a pathological trigger leading to neuronal injury, NFT formation and cell death which underpin neurodegeneration and cognitive decline in $\mathrm{AD}$ [8]. Recent evidence suggests that the failure 
or imbalance of A $\beta$ clearance, rather than APP mismetabolism, is the major contributor to toxic deposition in the sporadic form of the disease [25, 26]. This would attribute mechanisms implicated in the degradation of $\mathrm{A} \beta$ (i.e., neprilysin, insulin degrading enzyme (IDE), intracellular lysosomal degradation and microglia activation) of central importance in the development of $A \beta$ pathology [18, 27].

In recent years, with the amyloid cascade hypothesis as the driving force, the development of disease modifying $\mathrm{AD}$ therapeutics has focused on targeting the production or clearance of $\mathrm{A} \beta$ with the aim to inhibit its toxicity. These include; secretase inhibitors to reduce the production of $\mathrm{A} \beta$ fragment by APP mismetabolism, $A \beta$ aggregation inhibitors to prevent fibrillization and finally, active and passive immunotherapy which aim to capture soluble or aggregated $A \beta$ for clearance from the brain (reviewed in [28, 29]). Disappointingly, findings from Phase III passive immunotherapy trials targeting $A \beta$ have so far reported no cognitive benefit in mildto-moderate sporadic $\mathrm{AD}$. These worrying results have challenged the $A \beta$-centric theory, questioning if $\mathrm{A} \beta$ is simply a consequence and not a cause of $\mathrm{AD}$ pathogenesis. Nonetheless, these failures could also be attributed to logistical shortcomings; i) such trials recruited participants with established dementia, who are likely to be too far advanced in disease state to have a positive clinical benefit using this type of therapeutic; ii) recruitment criteria were purely based upon non-specific clinical assessments and therefore participants without the target pathology were likely to be included. In fact, the estimated contribution of $A \beta$-negative participants recruited into $A \beta$-targeted trials was estimated to be $>25 \%$, demonstrated by retrospective A $\beta$ PET imaging [30]. This highlights the importance for a biomarker-driven participant selection which will also have major impact on clinical decisions when prescribing A $\beta$-targeting drugs once these are available. At this time, on-going Phase III passive immunotherapy trials (Aducanumab, Crenezumab, Gantenerumab and Solanezumab) are targeting preclinical and/or mild $\mathrm{AD}$ with all participants recruited with supporting $\mathrm{A} \beta$ biomarker evidence - these trials reach completion during 2019 and 2020. An additional application of $\mathrm{A} \beta$ biomarkers would be their implementation into proof-of-principle Phase I trials [31] and/or proof-of-concept Phase II clinical trials [32] to assist in the triage of potential drug candidates. Some researchers would point towards the premature acceleration of poor drug candidates as a significant contributor to the number of failures in AD therapeutics. It has been commonplace to examine the capabilities of an $A \beta$ reducing molecule in transgenic $A D$ mice models, and if successful, examine said drug in large and expensive clinical trials without 
sufficient evidence of target engagement in humans. Consequently, biomarkers that detect and monitor biochemical effects of the drug are common in the early development of an AD drug [30].

\section{Imaging Biomarkers for A $\beta$ pathology}

The distinctive strength of $A \beta$-imaging across a clinical spectrum is the spatial nature of the quantitative information it provides, which fluid biomarkers simply cannot offer [33]. The development of Pittsburgh Compound B (PiB), a derivate of thioflavin-T, for the in vivo assessment of $\mathrm{A} \beta$ pathology, transformed the approach to $\mathrm{AD}$ in both a clinical and research setting allowing for significantly more advanced investigations. With a high affinity to fibrillar $\mathrm{A} \beta$ plaques, carbon-11 $\left({ }^{11} \mathrm{C}\right)$-labelled $\mathrm{PiB}$ has long been the most utilised $\mathrm{A} \beta$ tracer $[34,35]$. The major disadvantage of an ${ }^{11} \mathrm{C}$-labelled tracer, however, is the 20-minute half-life of the positron emitting isotope, limiting its use to centres with an onsite cyclotron. The advancement of "second generation" fluorine-18 $\left({ }^{18} \mathrm{~F}\right)$-labelled ligands, with a half-life of ${ }^{18} \mathrm{~F}$ of 110 minutes, has fostered wider availability for A $\beta$ PET, allowing for centralised synthesis and regional distribution. Encouragingly, $\mathrm{A} \beta$ specific ${ }^{18} \mathrm{~F}$ tracers have replicated findings from ${ }^{11} \mathrm{C}$ $\mathrm{PiB}$ studies in $\mathrm{AD}$ [36-39]. To date, three ${ }^{18} \mathrm{~F} \mathrm{~A} \beta$ specific radiotracers have been approved by the FDA and EMA for clinical use; ${ }^{18} \mathrm{~F}$-flutemetamol (Vizamy ${ }^{\circledR}$ ), ${ }^{18} \mathrm{~F}$-florbetapir (Amyvid ${ }^{\circledR}$ ) and ${ }^{18} \mathrm{~F}$-florbetapen $\left(\right.$ Neurace $\left.{ }^{\circledR}\right)$. Criticisms of the ${ }^{18} \mathrm{~F}$ tracers are an apparent inferior corticalto-white matter ratio compared with ${ }^{11} \mathrm{C}-\mathrm{PiB}$ and this has been attributed to a reduced overall cortical retention and higher non-specific white matter binding [40]. In preliminary studies, another (“third generation") A $\beta$ PET tracer, ${ }^{18}$ F-NAV4694 (formerly known as AZD-4694), has demonstrated the ability to visualise small $A \beta$ cortical deposits at the early stage of the disease due to reduced white matter binding, comparable to ${ }^{11} \mathrm{C}-\mathrm{PiB}$, but with the advantage of a longer half-life. Furthermore, ${ }^{18} \mathrm{~F}-\mathrm{NAV} 4694$ and ${ }^{11} \mathrm{C}-\mathrm{PiB}$ have been shown to have identical retention ranges and binding kinetics with an excellent correlation in cortical retention [40].

$\mathrm{A} \beta$ imaging has evolved from being exclusively a research tool to be integrated into clinical criteria for $\mathrm{AD}[26,41]$, mild cognitive impairment (MCI) [42] and preclinical AD [26] plus at the forefront of anti-A $\beta$ trials. Assistance from A $\beta$ PET has ensured superior subject selection, assessing target binding and evaluating treatment response of participants [30]. Inevitably, the use of several different $A \beta$ tracers across trials, clinics and research centres has highlighted a 
multi-centre issue. The "Centiloid-scale" for the conversion of semi-quantitative estimates in A $\beta$ PET imaging has been developed to produce a common quantitative output value to improve the application and comparison of $\mathrm{A} \beta$ imaging across different tracers. Currently, all ${ }^{18} \mathrm{~F}$ tracers are being cross calibrated against ${ }^{11} \mathrm{C}-\mathrm{PiB}[43]$.

\section{A $\beta$ imaging in Alzheimer's disease}

Visually assessed and quantitative PET studies that use diverse A $\beta$ tracers have consistently verified an increase in retention between $\mathrm{AD}$ and elderly controls, with the binding typically elevated in the frontal, cingulate, precuneus, striatum, parietal and lateral temporal cortices [12, 44-46]. Often the occipital, sensorimotor and mesial temporal cortices have a tendency to be less effected [45]. This regional distribution visualised by A $\beta$ PET retention closely follows the sequence of $\mathrm{A} \beta$ burden highlighted by post mortem studies [47-49]. However, owing to the long delay between the onset of disease pathophysiology and clinical manifestation, it has been difficult to ascertain the earliest regions of $A \beta$ deposition. Neuropathological post mortem staging of $A \beta$ phases demonstrate the frontal, parietal, temporal and occipital lobes occupying the preliminary stages of deposition in $\mathrm{AD}$ [50]. On the other hand, studies employing PET have demonstrated that the temporo-parietal junction and the precuneus are regions of early $A \beta$ accumulation, while the lateral and inferior temporal cortex are proposed for monitoring $\mathrm{A} \beta$ deposition in the symptomatic stages [45]. More recently, hierarchical regional staging of $\mathrm{A} \beta$ deposition further suggested that the temporobasal and frontomedial areas are first affected [51]. To address this long-standing question, Palmqvist and colleagues [52] identified regions of early accumulation by the novel stratification of individuals by both A $\beta$ PET and CSF biomarkers. Cross-sectional studies had previously indicated that CSF A $\beta 42$ changes precede changes in A $\beta$ PET standard uptake value ratios (SUVR) [53-56] with a recent longitudinal data adding further confirmation [57]. This signifies that individuals with AD may be initially classified as "CSF+/PET-" before converting to "CSF+/PET+" at a later stage. Using this methodology, a significant increase in $A \beta$ accumulation was observed in the precuneus, posterior cingulate cortex and orbitofrontal cortex when examining individuals with early accumulation (CSF+/PET-) against no measurable A $\beta$ accumulation (CSF-/PET-) [52]. Furthermore, increased retention in the medial orbitofrontal and posterior cingulate cortex at an even earlier stage was observed in individuals who converted from CSF-/PET- to $\mathrm{CSF}+$ PET- within two years in comparison to stable CSF-/PET-. This study specifically highlights regions within default mode network (DMN) and to a lesser extent the frontoparietal 
network as being associated with the very earliest signs of $A \beta$ accumulation [52] but more generally centres in the brain with higher levels of connectivity. $A \beta$ accumulation within the DMN has been previously reported in cross-sectional studies [58, 59] and it is suggested that enhanced activity or the metabolic demand of neurones located within the DMN exacerbate $\mathrm{A} \beta$ deposition [52]. Longitudinal studies of asymptomatic mutation carriers (PSEN1 and APP) have consistently demonstrated initial $\mathrm{A} \beta$ depositions occur distinctly in the striatum, which is not observed in sporadic AD [60-62].

There are rare exceptions to the general rule of increased A $\beta$ PET retention in AD (e.g. Arctic APP $[A P P$ arc $]$ mutation carriers). When considering autosomal-dominant AD (ADAD) mutation carriers, most cortical regions demonstrate increased PET retention except for the sensorimotor cortex $[60,61,63-66]$. It has been revealed that carriers of APParc mutation do not have ligand retention as shown by low ${ }^{11} \mathrm{C}-\mathrm{PiB}$ binding $[67,68]$ despite having $\mathrm{A} \beta$ pathology and positive AD-typical biomarkers (glucose metabolism, medial temporal lobe atrophy, and CSF A $\beta$ and tau changes) are all present in the same subjects [68]. The absence of cortical ${ }^{11} \mathrm{C}-\mathrm{PiB}$ retention is suggested to be caused by the amplified promotion of protofibril production due to a glutamic acid substitution in the $A \beta$ peptide [69]. Consequently, neuropathological assessment of $A P P$ arc mutation carriers demonstrated a lack of $A \beta$ densecore plaques which would preclude positive $A \beta$-imaging [68]. These studies further support the notion that $A \beta$ oligomers, not $A \beta$ fibrils, are the pathogens that inflict synaptic damage and cognitive decline. The presence of the apolipoprotein $\mathrm{E}(A P O E) \& 4$ allele is the most common genetic risk connected to sporadic $\mathrm{AD}$ and has been linked to earlier age of onset and a gene dose-dependent higher risk of developing $\mathrm{AD}[70,71]$. ApoE plays a role in $\mathrm{A} \beta$ metabolism and independent of clinical diagnosis $A P O E \varepsilon 4$ carriers present substantially higher $\mathrm{A} \beta$ deposition than non-carriers at an earlier age $[72,73]$. Despite this relationship, APOE $\varepsilon 4$ dosage does not affect the rate $\mathrm{A} \beta$ deposition over-time [13].

In the MCI population, $A \beta$ imaging has shown that $50-70 \%$ display $A D$ pathology $[74,75]$. In several studies, a non-amnestic MCI diagnosis has consistently low A $\beta$ binding and therefore a non-AD phenotype [12]. While there is a general agreement that $\mathrm{A} \beta$ burden only displays a weak correlation with memory impairment or disease severity, MCI patients demonstrate a consistent link between elevated $\mathrm{A} \beta$ retention and poorer episodic memory performance [76, 77]. 
In the healthy ageing population, it has been repeatedly shown that 25-35\% exhibit A $\beta$ PET SUVRs considered to be elevated $A \beta$ burden, predominately in the prefrontal and posterior cingulate regions [78-80]. This figure is similar to what was previously reported by earlier post mortem studies [81, 82]. However, despite being cognitively normal, the presence of elevated $\mathrm{A} \beta$ burden put this group at far greater risk to disease conversion than individuals with low $\mathrm{A} \beta$ PET SUVRs $[83,84]$. In fact, longitudinal A $\beta$ imaging studies have shown that the probability of cognitively unimpaired individuals with low $\mathrm{A} \beta$ burden developing $\mathrm{AD}$ is exceptionally low $[12,85]$.

$\mathrm{A} \beta$ imaging has added conclusive evidence to indicate that $\mathrm{A} \beta$ deposition occurs decades before the clinical onset of $\mathrm{AD}[6,13]$ and that cognitively healthy individuals with high $\mathrm{A} \beta$ burden are at significant risk to cognitive decline $[40,86]$. It is this group of individuals that are the most likely to benefit from on-going therapies targeting $A \beta$ clearance or production to halt the progression of $\mathrm{AD}$ [26]. Yet, the significant minority who exhibit $\mathrm{A} \beta$ burden who are absent of cognitive impairment, together with lack of strong relationship between $A \beta$ burden (by PET) with cognition and atrophy in $\mathrm{AD}$ suggests that $\mathrm{A} \beta$ burden alone cannot cause neurodegeneration but indirectly via other processes such as the propagation of tau outside of the medial temporal lobe [87]. In addition to this, $A \beta$ imaging which measures the fibrillar deposits may not correlate with cognitive decline as it has been suggested that soluble $A \beta$ exists as the neurotoxic form.

\section{$A \beta$ imaging in other dementias}

$\mathrm{A} \beta$ imaging has also been applied to a wide range of dementia-related conditions. While frontotemporolobar degeneration (FTLD) and Creutzfeldt-Jakob disease (CJD) exhibit no A $\beta$ burden, a large number of dementia with Lewy bodies (DLB) patients (50-80\%) demonstrate a cortical $\mathrm{A} \beta$ deposition distribution pattern similar to $\mathrm{AD}$ in both post mortem [88] and in vivo A $\beta$ PET studies [89]. DLB patients with negative A $\beta$ scans have been neuropathologically confirmed to have low A $\beta$ burden [90]. Commonality in early cognitive signs with AD makes a differential diagnosis of DLB difficult and with a mixed DLB/AD pathology commonly found at post mortem, A $\beta$ PET may be only useful in identifying the relatively rare pure DLB cases [91]. CAA is distinguished by localised $\mathrm{A} \beta$ deposition in small arterioles of the cerebral 
cortex. $\mathrm{A} \beta{ }^{11} \mathrm{C}-\mathrm{PiB}$ has demonstrated a posterior retention in $\mathrm{CAA}$ individuals that is distinct from a pattern typically observed in sporadic $\mathrm{AD}$ [92]. Further to this, focal A $\beta$ deposition has been shown to be helpful in highlighting new posterior microhaemorrhages that are usefully attributed to CAA patients [93]. Cortical A $\beta$ is not usually consistent with non-dementia Parkinson's (PD), with no A $\beta$ PET retention observed; conversely when dementia is present with PD (PDD) both vascular and cortical A $\beta$ deposition are present. A $\beta$ PET scans have been shown to determine which idiopathic normal-pressure hydrocephalus (iNPH) patients will benefit from shunt surgery by discriminating concomitant AD [94]. With A $\beta$ not being a pathological trait in FTLD and the clinical onset of the disease being similar to other dementias, A $\beta$ PET has been useful in the differential diagnosis between FTLD, different FTLD aphasias and $\mathrm{AD}$ [95]. Furthermore, the diagnostic performance of $\mathrm{A} \beta$ imaging has proven to be more accurate than FDG when diagnosing FTLD [96].

\section{Cerebrospinal Fluid (CSF) Biomarkers for $A \beta$ pathology, including $A \beta$ response markers}

The best-established fluid biomarkers for AD are CSF concentrations of total tau (T-tau), phosphorylated-tau (P-tau) and A $\beta 42$ [97]. Consistently, a marked increase in both T-tau and P-tau in AD, accompanied by a decrease in CSF A 342 (reflecting deposition of the protein in the brain parenchyma) has been found [97]. In the heterogenous MCI population (where approximately 50\% have AD) it has been shown that CSF A $\beta 42$ changes (coupled with T-tau and P-tau changes) have already occurred in those individuals who progress to clinical AD [98]. Importantly, CSF A $\beta$ is stable at follow-up when patients have reached dementia stage, indicating that $\mathrm{A} \beta$ levels do not change during the clinical phases of the disease. Hansson and colleagues [99], demonstrated that cognitively stable MCI patients do not have the typical AD biomarker profile, while progressive MCI patients could be identified with $95 \%$ sensitivity and 92\% specificity against elderly controls and $83 \%$ specificity against stable MCI cases. Further to this, longitudinal studies in presymptomatic individuals have demonstrated that CSF A $\beta 42$ changes are first detectable in the middle aged (45-54 years) and this is associated with later A $\beta$ PET positivity and cognitive decline [100].During the past five years, it has been confirmed that CSF $A \beta 42$ indeed is a reliable marker of $A \beta$ (senile plaque) pathology in the brain (as determined at autopsy or through A $\beta$ PET studies), especially when measured in a ratio with CSF $A \beta 40$ [101]. The CSF $A \beta 42 / A \beta 40$ ratio has been shown to have improved diagnostic 
accuracy for $\mathrm{A} \beta$ pathology in $\mathrm{AD}$ as $\mathrm{A} \beta 40$ acts as a normalization factor for total $\mathrm{A} \beta$ production, which otherwise could produce false-positives or false-negatives if not compensated for [102].

In addition, several byproducts of the proteolytic cleavage of APP, generating a variety of A $\beta$ species, can be measured in CSF [103-107]. However, these markers are still at preliminary investigation or show no association with $\mathrm{AD}$ while others have been shown to reflect target engagement in clinical trials $[108,109]$. For CSF T-tau and P-tau, the interpretation is less clear; tau markers are robustly increased in AD CSF [97], but the exact mechanism remains unclear [110]. Some data suggest that neurons exposed to Alzheimer-associated factors such as $A \beta$ may increase their secretion of both tau proteins [111]. Neurons who respond in this way may eventually accumulate tangle pathology and degenerate. In spite of these uncertainties, the diagnostic performance and clinical utility of CSF T-tau, P-tau and A $\beta 42$ are undisputed: new diagnostic algorithms including CSF biomarkers have been formulated [112], automated routine clinical chemistry assays for the markers are now becoming available [113] and standardization efforts to harmonize assays are well underway; reference methods for A $\beta 42$ have been formally certified by the Joint Committee for Traceability in Laboratory Medicine (JCTLM database accession numbers C11RMP9 and C12RMP1) [114, 115] and validated against amyloid PET [116], and a reference material for CSF A $\beta 42$ was recently released $\left(\right.$ ERM $^{\circledR}$-DA480/IFCC, ERM ${ }^{\circledR}$-DA481/IFCC and ERM ${ }^{\circledR}$-DA482/IFCC). Similar work is ongoing for CSF tau biomarkers.

\section{The concordance of CSF $A \beta$ and $A \beta$ PET}

There is now conclusive evidence as assessed in large cohorts with disease and age-matched controls that a good inverse correlation between A $\beta$ PET and CSF A $\beta 42$ exists (reviewed in [101]), while no correlation between A $\beta$ PET and CSF T-tau or P-tau has been reported [91]. Overall there is an agreement of $\sim 88 \%$ in classifying $A \beta+/$ - individuals, which is increased when assessing only clinically defined dementia (94\%). A positive CSF investigation in the absence of a negative A $\beta$ PET is more likely than the opposing. This is likely due to CSF changes occurring earliest and the disparity in measuring insoluble fibrillar $A \beta$ versus soluble diffusible $A \beta$ species $[100,117]$.

\section{Blood-based biomarkers for $A \beta$ pathology}


Both imaging biomarkers and CSF measurement of $\mathrm{A} \beta$ work well to identify $\mathrm{AD}$ pathophysiology. Nonetheless, PET imaging is costly, and access is limited to specialised centres. Therefore, it is seemingly unlikely to be implemented widely in a general routine assessment for cognitive complaints. CSF sampling is becoming routine in neurology clinics and the cost for the AD CSF assays are much lower per patient than for PET scans. For some clinicians, a lumbar puncture may be regarded as complicated, time-consuming and/or invasive. Therefore, a blood-based measure for $\mathrm{AD}$ or $\mathrm{AD}$ pathophysiology would have significant practical advantages for clinicians as well as being more attainable to patients.

It has been difficult to establish robust blood biomarkers for $\mathrm{A} \beta$ pathology in $\mathrm{AD}$. $\mathrm{A} \beta$ peptides can be measured in plasma but historically the correlation with $\mathrm{AD}$ and/or cerebral $\beta$ amyloidosis has been absent or weak (statistically significant but clinically meaningless) [97]. Plasma $A \beta$ concentrations have been interpreted as potentially influenced by production in platelets and other extra-cerebral tissues and the measurements have been confounded by matrix effects from plasma proteins [118]. Furthermore, the vast majority of peripheral A $\beta$ studies have concentrated on plasma/serum which do not take into account the substantial amounts of peptides bound to plasma proteins and blood cells [119]. However, this view is now starting to change. Recent mass spectrometric studies suggest that a ratio of a certain APP fragment (APP669-711) to $A \beta 42$ or $A \beta 42 / A \beta 40$ identifies $A \beta$-positive individuals with high sensitivity and specificity [120-122]. Nakamura and colleagues have described the accuracy of predicting ${ }^{11} \mathrm{C}-\mathrm{PiB}$ in the $\mathrm{AD} / \mathrm{MCI}$ and cognitively normal population at $91 \%$ and $87 \%$, respectively [122]. These results are in line with earlier data obtained using the ultrasensitive Simoa technology by which the sample can be diluted to remove confounding matrix effects in the $A \beta$ measurement [123]. In this study it was also shown that plasma $A \beta 42$ was decreased in $\mathrm{AD}$ compared with MCI, subjective cognitive decline (SCD) and controls, whereas, the $\mathrm{A} \beta 42 / \mathrm{A} \beta 40$ ratio could distinguish MCI from controls (please add ref here as well to make it very clear).

The most promising blood candidate for neurodegeneration to date is neurofilament light (NFL), which has shown to be reflective of CSF NFL $[124,125]$ and to be elevated in AD [125]. In addition, plasma NFL has been shown to be highest in AD and MCI cases with positive A $\beta$ PET scans and reduced A $\beta 42$ CSF biomarkers changes [125]. However, as NFL 
concentration also correlates with A $\beta$-independent conditions (poor cognition, MRI atrophy and NFT pathology) and is increased in non-AD neurodegenerative diseases [126, 127], it is likely that plasma NFL is a measure of generalised and on-going neurodegeneration, not of $\mathrm{A} \beta$ pathology per se. Nonetheless, as the amyloid cascade hypothesis suggests that $\mathrm{A} \beta$ deposition is the main initiator behind events that result in neurodegeneration, therefore a clear link between elevations of NFL in response to $\mathrm{A} \beta$ burden, if present, can be made.

In the advent of large well characterized research cohorts that now include A $\beta$ PET and/or CSF $\mathrm{A} \beta$ measures as routine, the opportunity to use an "endophenotype" approach to discover peripheral markers of $\mathrm{A} \beta$ pathology is ever increasing. Pilot data suggest associations of the concentrations of a number of plasma proteins (e.g., pancreatic polypeptide Y, IgM, chemokine ligand 13, interleukin 17, vascular cell adhesion protein 1, $\alpha 2$-macroglobulin, apolipoprotein $\mathrm{A} 1$, fibrinogen gamma chain, interleukins and complement proteins) and metabolites with $\mathrm{A} \beta$ burden in the brain [128-134]. However, these data should be interpreted with some caution, as they are derived from multi-marker panels and as a mechanistic understanding of the associations is currently lacking. The majority of these endophenotype studies have been confounded by the imbalance of AD/MCI individuals in the $\mathrm{A} \beta+$ group. Only a small number of studies have focused purely on cognitively normal participants with $\mathrm{A} \beta$ burden $[128,131]$.

\section{Future perspectives}

Utilising the advantages $A \beta$ imaging - CSF measures of $\mathrm{A} \beta$ accumulation seem to precede global A $\beta$ PET changes (CSF+/PET-). However, spatial information of A $\beta$ imaging has recently shown to highlight regions of early accumulation that later demonstrate CSF biomarkers changes (CSF-/PET-) [52]. This finding should have practical implications for the enrolment of participants to anti-A $\beta$ trials where there is evidence of $A \beta$ accumulation (early CSF changes or even earlier regional PET changes) but before neurodegeneration has set in. At the present time ${ }^{11} \mathrm{C}-\mathrm{PiB}$, and analogues thereof, detect $\mathrm{A} \beta$ plaques, mainly consisting of insoluble fibrils of $A \beta$. Insoluble $A \beta$ does not correlate well with disease progression and soluble $A \beta$ is a better marker of disease status. Therefore, aggregates rather than $A \beta$ plaques have been the focus in recent diagnostics and therapeutics. This highlights the pressing need for an imaging agent that can visualise soluble A $\beta$ aggregates. Recently, Sehlin and colleagues [135] have successfully demonstrated, in two mouse models, the use of an 
antibody-based (mAb158) PET ligand selective for $A \beta$ protofibrils for brain imaging. This has a potential implication in a number of neurodegenerative disorders as it demonstrates the feasibility of antibody-based in vivo imaging of proteins aggregates for such as $\alpha$ synuclein, tau or TDP-43.

New biomarkers for $A \beta$ plaque pathology - whilst neurons secrete $\mathrm{A} \beta$ species starting at amino acid 1 (the BACE1 cleavage site at APP), plaques contain a lot of $\mathrm{A} \beta$ (most of which ends at amino acid 42) that is N-terminally truncated (e.g., A $34-42$ ) and pyroGlu-modified (e.g., pyroGluA $\beta 3-42$ or 11-42). Potentially, these $A \beta$ species form in the plaques and could serve as fluid-based biomarkers for cerebral $\mathrm{A} \beta$ pathology. Ultrasensitive assays could potentially trace such brain-derived $A \beta$ forms in the blood, which would solve the problem with contamination of the signal by A $\beta 1-42$ derived from extracerebral tissues. There are also many $\mathrm{A} \beta$ forms that are either being produced from APP or have been found in brain tissue that could be explored as potential $A \beta$ pathology or toxicity markers. These include $\mathrm{N}$-terminally extended $A \beta$ forms that span the BACE1 cleavage site of APP $[103,136-138]$ and $A \beta$ forms that extend C-terminally towards the $\delta$ - and $\varepsilon$-cleavage sites at amino acids 45, 46, 48 and 49 [139]. As no reliable assays presently exist to measure these $A \beta$ forms to assess their pathophysiological relevance and relation to disease state, they were jokingly referred to as "the dark matter of $A \beta$ " at the Clinical Trials in Alzheimer's disease (CTAD) conference in 2017.

Blood-based challenges and approaches - There are considerable challenges ahead in developing a blood-based marker that can accurately reflect $A \beta$ pathology and working groups are taking steps, taking lessons from the CSF community, to standardize pre-analytical variables [140]. Without a doubt, blood measures of tau and NFL are the leading blood-based candidates for $\mathrm{AD}$ but the view-point on peripheral $\mathrm{A} \beta$ species is changing and should be revisited. The constant evolution of sensitive instrumentation targeting single putative candidates, guided by unbiased discovery methodologies, mean the prospect of clinically meaningful blood-based biomarker for $\mathrm{A} \beta$ pathology and/or $\mathrm{AD}$ is a genuine possibility. Novel targets that associate with $A \beta$ plaque pathology have been suggested in abundance and should be taken with caution whilst a mechanistic link can be established. To date, blood-based studies using an $A \beta$ pathology endophenotype design have almost exclusively utilised global 
measures of $A \beta$-imaging as a surrogate marker. With recent evidence demonstrating the earliest signs of $A \beta$ accumulation to be visible in the CSF [57] or regional specific PET [52], such studies should now be adopting these modalities as more accurate endophenotypes of early A $\beta$ accumulation.

\section{Executive summary}

\section{$\mathrm{A} \beta$ in Alzheimer's disease}

- The "amyloid cascade hypothesis", has been the prevailing theory suggesting that $\mathrm{A} \beta$ aggregation acts as a pathological trigger leading to neurodegeneration.

- Development of $\mathrm{AD}$ therapeutics have focused on targeting the production or clearance of $A \beta$ with the aim to inhibit its toxicity, prevent plaque build-up and/or plaque clearance.

- Accurate $A \beta$ imaging and fluid biomarkers are critical to support disease-specific trials in participant selection and proof of target engagement

\section{Imaging Biomarkers for $A \beta$ pathology}

- $\mathrm{A} \beta\left({ }^{11} \mathrm{C}\right.$ and $\left.{ }^{18} \mathrm{~F}\right)$ PET imaging has added conclusive evidence that $\mathrm{A} \beta$ deposition occurs decades before the clinical onset of AD. A $\beta$ PET studies have consistently found an increase in retention between $\mathrm{AD}, \mathrm{MCI}$ and elderly controls.

- Regions within default mode network (DMN) have been shown to be the very earliest cortical area of $A \beta$ accumulation, even preceding CSF A $\beta$ changes.

Cerebrospinal Fluid (CSF) Biomarkers for $A \beta$ pathology, including $A \beta$ response markers

- A decreased CSF $A \beta 42$ is a reliable marker of $A \beta$ (senile plaque) pathology and the CSF $A \beta 42 / A \beta 40$ further increases diagnostic accuracy for $A \beta$ pathology in AD.

- CSF A 342 positive biomarkers precede a positive global PET SUVR signal.

- Validated reference material for CSF A 342 has been certified and is now available.

\section{Blood-based biomarkers for $A \beta$ pathology}

- $\mathrm{A} \beta$ species in blood have been widely shown to be not clinically relevant however; the measurement of the APP669-711/A $\beta 42$ ratio has been shown to be predictive of $\mathrm{A} \beta$ plaque pathology with high accuracy.

Future perspectives 
- Fluid-based ultrasensitive assays that target novel A $\beta$ species found in plaques $(\mathrm{N}$ terminally truncated or post-translational modified) could serve as more accurate markers $\mathrm{A} \beta$ pathology (particularly in blood).

- Better endophenotype stratification (regional PET or CSF A $\beta 42$ ) may foster improved and early peripheral panels for $A \beta$ burden.

\section{Financial disclosures and acknowledgements}

$\mathrm{KB}$ and $\mathrm{HZ}$ are co-founders of Brain Biomarker Solutions in Gothenburg AB, a GU Venturesbased platform company at the University of Gothenburg. HZ has served at advisory boards of Eli Lilly and Roche Diagnostics and has received travel support from TEVA. KB has served as a consultant or at advisory boards for Alzheon, Eli Lilly, Fujirebio Europe, I.B.L. International, Novartis, and Roche Diagnostics. MS has received funding from the Knut och Alice Wallenberg Foundation, the Wallenberg Centre for Molecular and Translational Medicine, and the Swedish Research Council.

\section{References}

1. Prince, M., et al., The global prevalence of dementia: a systematic review and metaanalysis. Alzheimers Dement, 2013. 9(1): p. 63-75 e2.

2. $\quad$ Scheltens, P., et al., Alzheimer's disease. Lancet, 2016. 388(10043): p. 505-17.

3. Masters, C.L., et al., Amyloid plaque core protein in Alzheimer disease and Down syndrome. Proc Natl Acad Sci U S A, 1985. 82(12): p. 4245-9.

4. Van Cauwenberghe, C., C. Van Broeckhoven, and K. Sleegers, The genetic landscape of Alzheimer disease: clinical implications and perspectives. Genet Med, 2016. 18(5): p. 421-30.

5. Selkoe, D.J., Soluble oligomers of the amyloid beta-protein impair synaptic plasticity and behavior. Behav Brain Res, 2008. 192(1): p. 106-13.

6. Jack, C.R., Jr., et al., Tracking pathophysiological processes in Alzheimer's disease: an updated hypothetical model of dynamic biomarkers. Lancet Neurol, 2013. 12(2): p. 207-16.

7. De Strooper, B. and E. Karran, The Cellular Phase of Alzheimer's Disease. Cell, 2016. 164(4): p. 603-15.

8. Hardy, J.A. and G.A. Higgins, Alzheimer's disease: the amyloid cascade hypothesis. Science, 1992. 256(5054): p. 184-5.

9. Selkoe, D.J. and J. Hardy, The amyloid hypothesis of Alzheimer's disease at 25 years. EMBO Mol Med, 2016. 8(6): p. 595-608.

10. Chetelat, G., Reply: The amyloid cascade is not the only pathway to AD. Nat Rev Neurol, 2013. 9(6): p. 356.

11. Pimplikar, S.W., Reassessing the amyloid cascade hypothesis of Alzheimer's disease. Int J Biochem Cell Biol, 2009. 41(6): p. 1261-8.

12. Villemagne, V.L., et al., Longitudinal assessment of Abeta and cognition in aging and Alzheimer disease. Ann Neurol, 2011. 69(1): p. 181-92. 
13. Villemagne, V.L., et al., Amyloid beta deposition, neurodegeneration, and cognitive decline in sporadic Alzheimer's disease: a prospective cohort study. Lancet Neurol, 2013. 12(4): p. 357-67.

14. Dubois, B., et al., Advancing research diagnostic criteria for Alzheimer's disease: the IWG-2 criteria. Lancet Neurol, 2014. 13(6): p. 614-29.

15. Villemagne, V.L., et al., Abeta-amyloid and Tau Imaging in Dementia. Semin Nucl Med, 2017. 47(1): p. 75-88.

16. Zhang, Y.W., et al., APP processing in Alzheimer's disease. Mol Brain, 2011. 4: p. 3.

17. Chow, V.W., et al., An overview of APP processing enzymes and products. Neuromolecular Med, 2010. 12(1): p. 1-12.

18. Murphy, M.P. and H. LeVine, 3rd, Alzheimer's disease and the amyloid-beta peptide. J Alzheimers Dis, 2010. 19(1): p. 311-23.

19. Masters, C.L., et al., Molecular mechanisms for Alzheimer's disease: implications for neuroimaging and therapeutics. J Neurochem, 2006. 97(6): p. 1700-25.

20. Iwatsubo, T., et al., Visualization of A beta 42(43) and A beta 40 in senile plaques with end-specific A beta monoclonals: evidence that an initially deposited species is A beta 42(43). Neuron, 1994. 13(1): p. 45-53.

21. Walsh, D.M. and D.J. Selkoe, A beta oligomers - a decade of discovery. J Neurochem, 2007. 101(5): p. 1172-84.

22. Ballard, C., et al., Alzheimer's disease. Lancet, 2011. 377(9770): p. 1019-31.

23. Eikelenboom, P., et al., Inflammatory mechanisms in Alzheimer's disease. Trends Pharmacol Sci, 1994. 15(12): p. 447-50.

24. McGeer, P.L. and E.G. McGeer, The inflammatory response system of brain: implications for therapy of Alzheimer and other neurodegenerative diseases. Brain Res Brain Res Rev, 1995. 21(2): p. 195-218.

25. Mawuenyega, K.G., et al., Decreased clearance of CNS beta-amyloid in Alzheimer's disease. Science, 2010. 330(6012): p. 1774.

26. Sperling, R.A., et al., Toward defining the preclinical stages of Alzheimer's disease: recommendations from the National Institute on Aging-Alzheimer's Association workgroups on diagnostic guidelines for Alzheimer's disease. Alzheimers Dement, 2011. 7(3): p. 280-92.

27. Agostinho, P., R.A. Cunha, and C. Oliveira, Neuroinflammation, oxidative stress and the pathogenesis of Alzheimer's disease. Curr Pharm Des, 2010. 16(25): p. 2766-78.

28. Jindal, H., et al., Alzheimer disease immunotherapeutics: then and now. Hum Vaccin Immunother, 2014. 10(9): p. 2741-3.

29. Folch, J., et al., Current Research Therapeutic Strategies for Alzheimer's Disease Treatment. Neural Plast, 2016. 2016: p. 8501693.

30. Salloway, S., et al., Two phase 3 trials of bapineuzumab in mild-to-moderate Alzheimer's disease. N Engl J Med, 2014. 370(4): p. 322-33.

31. Blennow, K., H. Hampel, and H. Zetterberg, Biomarkers in amyloid-beta immunotherapy trials in Alzheimer's disease. Neuropsychopharmacology, 2014. 39(1): p. 189-201.

32. Lannfelt, L., et al., Safety, efficacy, and biomarker findings of PBT2 in targeting Abeta as a modifying therapy for Alzheimer's disease: a phase IIa, double-blind, randomised, placebo-controlled trial. Lancet Neurol, 2008. 7(9): p. 779-86.

33. Johnson, K.A., et al., Brain imaging in Alzheimer disease. Cold Spring Harb Perspect Med, 2012. 2(4): p. a006213.

34. Cohen, A.D., et al., Using Pittsburgh Compound B for in vivo PET imaging of fibrillar amyloid-beta. Adv Pharmacol, 2012. 64: p. 27-81. 
35. Klunk, W.E., et al., Imaging brain amyloid in Alzheimer's disease with Pittsburgh Compound-B. Ann Neurol, 2004. 55(3): p. 306-19.

36. Nelissen, N., et al., Phase 1 study of the Pittsburgh compound B derivative 18Fflutemetamol in healthy volunteers and patients with probable Alzheimer disease. $\mathrm{J}$ Nucl Med, 2009. 50(8): p. 1251-9.

37. Vandenberghe, R., et al., Amyloid PET in clinical practice: Its place in the multidimensional space of Alzheimer's disease. Neuroimage Clin, 2013. 2: p. 497-511.

38. Choi, S.R., et al., Preclinical properties of 18F-AV-45: a PET agent for Abeta plaques in the brain. J Nucl Med, 2009. 50(11): p. 1887-94.

39. Wolk, D.A., et al., Amyloid imaging in Alzheimer's disease: comparison of florbetapir and Pittsburgh compound-B positron emission tomography. J Neurol Neurosurg Psychiatry, 2012. 83(9): p. 923-6.

40. Rowe, C.C., et al., Head-to-head comparison of 11C-PiB and 18F-AZD4694 (NAV4694) for beta-amyloid imaging in aging and dementia. J Nucl Med, 2013. 54(6): p. 880-6.

41. Dubois, B., et al., Revising the definition of Alzheimer's disease: a new lexicon. Lancet Neurol, 2010. 9(11): p. 1118-27.

42. Albert, M.S., et al., The diagnosis of mild cognitive impairment due to Alzheimer's disease: recommendations from the National Institute on Aging-Alzheimer's Association workgroups on diagnostic guidelines for Alzheimer's disease. Alzheimers Dement, 2011. 7(3): p. 270-9.

43. Klunk, W.E., et al., The Centiloid Project: standardizing quantitative amyloid plaque estimation by PET. Alzheimers Dement, 2015. 11(1): p. 1-15 e1-4.

44. Jack, C.R., Jr., J.R. Barrio, and V. Kepe, Cerebral amyloid PET imaging in Alzheimer's disease. Acta Neuropathol, 2013. 126(5): p. 643-57.

45. Villain, N., et al., Regional dynamics of amyloid-beta deposition in healthy elderly, mild cognitive impairment and Alzheimer's disease: a voxelwise PiB-PET longitudinal study. Brain, 2012. 135(Pt 7): p. 2126-39.

46. Scheinin, N.M., et al., Follow-up of [11C]PIB uptake and brain volume in patients with Alzheimer disease and controls. Neurology, 2009. 73(15): p. 1186-92.

47. Braak, H. and E. Braak, Frequency of stages of Alzheimer-related lesions in different age categories. Neurobiol Aging, 1997. 18(4): p. 351-7.

48. Ikonomovic, M.D., et al., Post-mortem correlates of in vivo PiB-PET amyloid imaging in a typical case of Alzheimer's disease. Brain, 2008. 131(Pt 6): p. 1630-45.

49. Kadir, A., et al., Positron emission tomography imaging and clinical progression in relation to molecular pathology in the first Pittsburgh Compound B positron emission tomography patient with Alzheimer's disease. Brain, 2011. 134(Pt 1): p. 301-17.

50. Thal, D.R., et al., Phases of A beta-deposition in the human brain and its relevance for the development of AD. Neurology, 2002. 58(12): p. 1791-800.

51. Grothe, M.J., et al., In vivo staging of regional amyloid deposition. Neurology, 2017. 89(20): p. 2031-2038.

52. Palmqvist, S., et al., Earliest accumulation of beta-amyloid occurs within the defaultmode network and concurrently affects brain connectivity. Nat Commun, 2017. 8(1): p. 1214.

53. Bateman, R.J., et al., Clinical and biomarker changes in dominantly inherited Alzheimer's disease. N Engl J Med, 2012. 367(9): p. 795-804.

54. Blennow, K., H. Zetterberg, and A.M. Fagan, Fluid biomarkers in Alzheimer disease. Cold Spring Harb Perspect Med, 2012. 2(9): p. a006221. 
55. Fagan, A.M., et al., Cerebrospinal fluid tau and ptau(181) increase with cortical amyloid deposition in cognitively normal individuals: implications for future clinical trials of Alzheimer's disease. EMBO Mol Med, 2009. 1(8-9): p. 371-80.

56. Mattsson, N., et al., Independent information from cerebrospinal fluid amyloid-beta and florbetapir imaging in Alzheimer's disease. Brain, 2015. 138(Pt 3): p. 772-83.

57. Palmqvist, S., N. Mattsson, and O. Hansson, Cerebrospinal fluid analysis detects cerebral amyloid-beta accumulation earlier than positron emission tomography. Brain, 2016. 139(Pt 4): p. 1226-36.

58. Sperling, R.A., et al., Amyloid deposition is associated with impaired default network function in older persons without dementia. Neuron, 2009. 63(2): p. 178-88.

59. Myers, N., et al., Within-patient correspondence of amyloid-beta and intrinsic network connectivity in Alzheimer's disease. Brain, 2014. 137(Pt 7): p. 2052-64.

60. Klunk, W.E., et al., Amyloid deposition begins in the striatum of presenilin-1 mutation carriers from two unrelated pedigrees. J Neurosci, 2007. 27(23): p. 6174-84.

61. Villemagne, V.L., et al., High striatal amyloid beta-peptide deposition across different autosomal Alzheimer disease mutation types. Arch Neurol, 2009. 66(12): p. 1537-44.

62. Koivunen, J., et al., PET amyloid ligand [11C]PIB uptake shows predominantly striatal increase in variant Alzheimer's disease. Brain, 2008. 131(Pt 7): p. 1845-53.

63. Benzinger, T.L., et al., Regional variability of imaging biomarkers in autosomal dominant Alzheimer's disease. Proc Natl Acad Sci U S A, 2013. 110(47): p. E4502-9.

64. Knight, W.D., et al., Carbon-11-Pittsburgh compound B positron emission tomography imaging of amyloid deposition in presenilin 1 mutation carriers. Brain, 2011. 134(Pt 1): p. 293-300.

65. Remes, A.M., et al., Carbon 11-labeled pittsburgh compound B positron emission tomographic amyloid imaging in patients with APP locus duplication. Arch Neurol, 2008. 65(4): p. 540-4.

66. Scholl, M., et al., Glucose metabolism and PIB binding in carriers of a His163Tyr presenilin 1 mutation. Neurobiol Aging, 2011. 32(8): p. 1388-99.

67. Tomiyama, T., et al., A new amyloid beta variant favoring oligomerization in Alzheimer's-type dementia. Ann Neurol, 2008. 63(3): p. 377-87.

68. Scholl, M., et al., Low PiB PET retention in presence of pathologic CSF biomarkers in Arctic APP mutation carriers. Neurology, 2012. 79(3): p. 229-36.

69. Nilsberth, C., et al., The 'Arctic' APP mutation (E693G) causes Alzheimer's disease by enhanced Abeta protofibril formation. Nat Neurosci, 2001. 4(9): p. 887-93.

70. Papassotiropoulos, A., et al., Genetics, transcriptomics, and proteomics of Alzheimer's disease. J Clin Psychiatry, 2006. 67(4): p. 652-70.

71. Farrer, L.A., et al., Effects of age, sex, and ethnicity on the association between apolipoprotein E genotype and Alzheimer disease. A meta-analysis. APOE and Alzheimer Disease Meta Analysis Consortium. JAMA, 1997. 278(16): p. 1349-56.

72. $\quad$ Ossenkoppele, R., et al., Prevalence of amyloid PET positivity in dementia syndromes: a meta-analysis. JAMA, 2015. 313(19): p. 1939-49.

73. Lautner, R., et al., Preclinical effects of APOE epsilon4 on cerebrospinal fluid Abeta42 concentrations. Alzheimers Res Ther, 2017. 9(1): p. 87.

74. Forsberg, A., et al., PET imaging of amyloid deposition in patients with mild cognitive impairment. Neurobiol Aging, 2008. 29(10): p. 1456-65.

75. Kemppainen, N.M., et al., PET amyloid ligand [11C]PIB uptake is increased in mild cognitive impairment. Neurology, 2007. 68(19): p. 1603-6.

76. Mormino, E.C., et al., Episodic memory loss is related to hippocampal-mediated betaamyloid deposition in elderly subjects. Brain, 2009. 132(Pt 5): p. 1310-23. 
77. Ong, K., et al., (18)F-florbetaben Abeta imaging in mild cognitive impairment. Alzheimers Res Ther, 2013. 5(1): p. 4.

78. Villemagne, V.L., et al., Abeta deposits in older non-demented individuals with cognitive decline are indicative of preclinical Alzheimer's disease. Neuropsychologia, 2008. 46(6): p. 1688-97.

79. Rowe, C.C., et al., Amyloid imaging results from the Australian Imaging, Biomarkers and Lifestyle (AIBL) study of aging. Neurobiol Aging, 2010. 31(8): p. 1275-83.

80. Aizenstein, H.J., et al., Frequent amyloid deposition without significant cognitive impairment among the elderly. Arch Neurol, 2008. 65(11): p. 1509-17.

81. Davies, L., et al., A4 amyloid protein deposition and the diagnosis of Alzheimer's disease: prevalence in aged brains determined by immunocytochemistry compared with conventional neuropathologic techniques. Neurology, 1988. 38(11): p. 1688-93.

82. Morris, J.C. and J.L. Price, Pathologic correlates of nondemented aging, mild cognitive impairment, and early-stage Alzheimer's disease. J Mol Neurosci, 2001. 17(2): p. 10118.

83. Sperling, R.A., et al., Amyloid deposition detected with florbetapir F 18 ((18)F-AV-45) is related to lower episodic memory performance in clinically normal older individuals. Neurobiol Aging, 2013. 34(3): p. 822-31.

84. Jagust, W., Is amyloid-beta harmful to the brain? Insights from human imaging studies. Brain, 2016. 139(Pt 1): p. 23-30.

85. Rowe, C.C., et al., Predicting Alzheimer disease with beta-amyloid imaging: results from the Australian imaging, biomarkers, and lifestyle study of ageing. Ann Neurol, 2013. 74(6): p. 905-13.

86. Resnick, S.M., et al., Longitudinal cognitive decline is associated with fibrillar amyloid-beta measured by [11C]PiB. Neurology, 2010. 74(10): p. 807-15.

87. Stancu, I.C., et al., Models of beta-amyloid induced Tau-pathology: the long and "folded" road to understand the mechanism. Mol Neurodegener, 2014. 9: p. 51.

88. Lippa, C.F., et al., DLB and PDD boundary issues: diagnosis, treatment, molecular pathology, and biomarkers. Neurology, 2007. 68(11): p. 812-9.

89. Gomperts, S.N., et al., Imaging amyloid deposition in Lewy body diseases. Neurology, 2008. 71(12): p. 903-10.

90. Ikonomovic, M.D., et al., Early AD pathology in a [C-11]PiB-negative case: a PiBamyloid imaging, biochemical, and immunohistochemical study. Acta Neuropathol, 2012. 123(3): p. 433-47.

91. Villemagne, V.L. and G. Chetelat, Neuroimaging biomarkers in Alzheimer's disease and other dementias. Ageing Res Rev, 2016. 30: p. 4-16.

92. Johnson, K.A., et al., Imaging of amyloid burden and distribution in cerebral amyloid angiopathy. Ann Neurol, 2007. 62(3): p. 229-34.

93. Farid, K., A. Charidimou, and J.C. Baron, Amyloid positron emission tomography in sporadic cerebral amyloid angiopathy: A systematic critical update. Neuroimage Clin, 2017. 15: p. 247-263.

94. Jang, H., et al., Prognostic value of amyloid PET scan in normal pressure hydrocephalus. J Neurol, 2017.

95. Rabinovici, G.D., et al., Abeta amyloid and glucose metabolism in three variants of primary progressive aphasia. Ann Neurol, 2008. 64(4): p. 388-401.

96. Rabinovici, G.D., et al., Amyloid vs FDG-PET in the differential diagnosis of AD and FTLD. Neurology, 2011. 77(23): p. 2034-42.

97. Olsson, B., et al., CSF and blood biomarkers for the diagnosis of Alzheimer's disease: a systematic review and meta-analysis. Lancet Neurol, 2016. 15(7): p. 673-684. 
98. Blennow, K., A Review of Fluid Biomarkers for Alzheimer's Disease: Moving from CSF to Blood. Neurol Ther, 2017. 6(Suppl 1): p. 15-24.

99. Hansson, O., et al., Association between CSF biomarkers and incipient Alzheimer's disease in patients with mild cognitive impairment: a follow-up study. Lancet Neurol, 2006. 5(3): p. 228-34.

100. Sutphen, C.L., et al., Longitudinal Cerebrospinal Fluid Biomarker Changes in Preclinical Alzheimer Disease During Middle Age. JAMA Neurol, 2015. 72(9): p. 1029-42.

101. Blennow, K., et al., Amyloid biomarkers in Alzheimer's disease. Trends Pharmacol Sci, 2015. 36(5): p. 297-309.

102. Lewczuk, P., et al., Amyloid-beta 42/40 cerebrospinal fluid concentration ratio in the diagnostics of Alzheimer's disease: validation of two novel assays. $\mathrm{J}$ Alzheimers Dis, 2015. 43(1): p. 183-91.

103. Brinkmalm, G., et al., An online nano-LC-ESI-FTICR-MS method for comprehensive characterization of endogenous fragments from amyloid beta and amyloid precursor protein in human and cat cerebrospinal fluid. J Mass Spectrom, 2012. 47(5): p. 591603.

104. Portelius, E., et al., Novel abeta isoforms in Alzheimer's disease - their role in diagnosis and treatment. Curr Pharm Des, 2011. 17(25): p. 2594-602.

105. Zetterberg, H., et al., Elevated cerebrospinal fluid BACE1 activity in incipient Alzheimer disease. Arch Neurol, 2008. 65(8): p. 1102-7.

106. Holtta, M., et al., Evaluating amyloid-beta oligomers in cerebrospinal fluid as a biomarker for Alzheimer's disease. PLoS One, 2013. 8(6): p. e66381.

107. Savage, M.J., et al., A sensitive abeta oligomer assay discriminates Alzheimer's and aged control cerebrospinal fluid. J Neurosci, 2014. 34(8): p. 2884-97.

108. Portelius, E., et al., beta-site amyloid precursor protein-cleaving enzyme 1(BACE1) inhibitor treatment induces Abeta5-X peptides through alternative amyloid precursor protein cleavage. Alzheimers Res Ther, 2014. 6(5-8): p. 75.

109. Portelius, E., et al., A novel Abeta isoform pattern in CSF reflects gamma-secretase inhibition in Alzheimer disease. Alzheimers Res Ther, 2010. 2(2): p. 7.

110. Zetterberg, H., Review: Tau in biofluids - relation to pathology, imaging and clinical features. Neuropathol Appl Neurobiol, 2017.

111. Maia, L.F., et al., Changes in Amyloid-beta and Tau in the Cerebrospinal Fluid of Transgenic Mice Overexpressing Amyloid Precursor Protein. Sci Transl Med, 2013. 5(194): p. 194re2.

112. Dubois, B., et al., Advancing research diagnostic criteria for Alzheimer's disease: the IWG-2 criteria. Lancet Neurol, 2014. 13(6): p. 614-629.

113. Bittner, T., et al., Technical performance of a novel, fully automated electrochemiluminescence immunoassay for the quantitation of beta-amyloid (1-42) in human cerebrospinal fluid. Alzheimers Dement, 2015.

114. Korecka, M., et al., Qualification of a surrogate matrix-based absolute quantification method for amyloid-beta(4)(2) in human cerebrospinal fluid using 2D UPLC-tandem mass spectrometry. J Alzheimers Dis, 2014. 41(2): p. 441-51.

115. Leinenbach, A., et al., Mass Spectrometry-Based Candidate Reference Measurement Procedure for Quantification of Amyloid-beta in Cerebrospinal Fluid. Clin Chem, 2014.

116. Pannee, J., et al., Reference measurement procedure for CSF amyloid beta (Abeta)1-42 and the CSF Abetal-42 /Abetal-40 ratio - a cross-validation study against amyloid PET. J Neurochem, 2016. 139(4): p. 651-658. 
117. Toledo, J.B., et al., Nonlinear Association Between Cerebrospinal Fluid and Florbetapir F-18 beta-Amyloid Measures Across the Spectrum of Alzheimer Disease. JAMA Neurol, 2015. 72(5): p. 571-81.

118. Zetterberg, H., Plasma amyloid beta-quo vadis? Neurobiol Aging, 2015. 36(10): p. 2671-3.

119. Pesini, P., et al., Reliable Measurements of the beta-Amyloid Pool in Blood Could Help in the Early Diagnosis of AD. Int J Alzheimers Dis, 2012. 2012: p. 604141.

120. Kaneko, N., et al., Novel plasma biomarker surrogating cerebral amyloid deposition. Proc Jpn Acad Ser B Phys Biol Sci, 2014. 90(9): p. 353-64.

121. Ovod, V., et al., Amyloid beta concentrations and stable isotope labeling kinetics of human plasma specific to central nervous system amyloidosis. Alzheimers Dement, 2017.

122. Nakamura, A., et al., High performance plasma amyloid-beta biomarkers for Alzheimer's disease. Nature, 2018. 554(7691): p. 249-254.

123. Janelidze, S., et al., Plasma beta-amyloid in Alzheimer's disease and vascular disease. Sci Rep, 2016. 6: p. 26801.

124. Gisslen, M., et al., Plasma Concentration of the Neurofilament Light Protein (NFL) is a Biomarker of CNS Injury in HIV Infection: A Cross-Sectional Study. EBioMedicine, 2016. 3: p. 135-40.

125. Mattsson, N., et al., Association of Plasma Neurofilament Light With Neurodegeneration in Patients With Alzheimer Disease. JAMA Neurol, 2017. 74(5): p. 557-566.

126. Rohrer, J.D., et al., Serum neurofilament light chain protein is a measure of disease intensity in frontotemporal dementia. Neurology, 2016. 87(13): p. 1329-36.

127. Rojas, J., et al., Plasma neurofilament light chain predicts disease progression in progressive supranuclear palsy. Journal of Neurochemistry, 2016. 138: p. 241-242.

128. Thambisetty, M., et al., Proteome-based plasma markers of brain amyloid-beta deposition in non-demented older individuals. J Alzheimers Dis, 2010. 22(4): p. 1099109.

129. Voyle, N., et al., Blood Protein Markers of Neocortical Amyloid-beta Burden: A Candidate Study Using SOMAscan Technology. J Alzheimers Dis, 2015. 46(4): p. 94761.

130. Burnham, S.C., et al., Predicting Alzheimer disease from a blood-based biomarker profile: A 54-month follow-up. Neurology, 2016. 87(11): p. 1093-101.

131. Westwood, S., et al., Blood-Based Biomarker Candidates of Cerebral Amyloid Using PiB PET in Non-Demented Elderly. J Alzheimers Dis, 2016. 52(2): p. 561-72.

132. Ashton, N.J., et al., Blood protein predictors of brain amyloid for enrichment in clinical trials? Alzheimers Dement (Amst), 2015. 1(1): p. 48-60.

133. Pedrini, S., et al., A blood-based biomarker panel indicates IL-10 and IL-12/23p40 are jointly associated as predictors of beta-amyloid load in an AD cohort. Sci Rep, 2017. 7(1): p. 14057.

134. Voyle, N., et al., Blood metabolite markers of neocortical amyloid-beta burden: discovery and enrichment using candidate proteins. Transl Psychiatry, 2016. 6: p. e719.

135. Sehlin, D., et al., Antibody-based PET imaging of amyloid beta in mouse models of Alzheimer's disease. Nat Commun, 2016. 7: p. 10759.

136. Portelius, E., et al., Identification of novel APP/Abeta isoforms in human cerebrospinal fluid. Neurodegener Dis, 2009. 6(3): p. 87-94.

137. Portelius, E., et al., Mass spectrometric characterization of amyloid-beta species in the 7PA2 cell model of Alzheimer's disease. J Alzheimers Dis, 2013. 33(1): p. 85-93. 
138. Willem, M., et al., eta-Secretase processing of APP inhibits neuronal activity in the hippocampus. Nature, 2015. 526(7573): p. 443-7.

139. Lessard, C.B., et al., gamma-Secretase Modulators and APH1 Isoforms Modulate gamma-Secretase Cleavage but Not Position of epsilon-Cleavage of the Amyloid Precursor Protein (APP). PLoS One, 2015. 10(12): p. e0144758.

140. O'Bryant, S.E., et al., Guidelines for the standardization of preanalytic variables for blood-based biomarker studies in Alzheimer's disease research. Alzheimers Dement, 2015. 11(5): p. 549-60.

\section{Reference annotations}

** Palmqvist, S., N. Mattsson, and O. Hansson, Cerebrospinal fluid analysis detects cerebral amyloid-beta accumulation earlier than positron emission tomography. Brain, 2016. 139(Pt 4): p. 1226-36 - cerebrospinal fluid amyloid- $\beta 42$ becomes abnormal in the earliest stages of Alzheimer's disease, before amyloid positron emission tomography and before neurodegeneration starts.

**Palmqvist, S., et al., Earliest accumulation of beta-amyloid occurs within the default-mode network and concurrently affects brain connectivity. Nat Commun, 2017. 8(1): p. 1214 - A $\beta$ fibrils start to accumulate within certain parts of the DMN in preclinical AD before CSF A $\beta$ become present.

*Sutphen, C.L., et al., Longitudinal Cerebrospinal Fluid Biomarker Changes in Preclinical Alzheimer Disease During Middle Age. JAMA Neurol, 2015. 72(9): p. 1029-42 - Longitudinal CSF biomarker patterns consistent with AD are first detectable during early middle age and are associated with later amyloid positivity and cognitive decline

* Janelidze, S., et al., Plasma beta-amyloid in Alzheimer's disease and vascular disease. Sci Rep, 2016. 6: p. 26801 - Using the ultra senstive digital ELISA (Simoa) plasma levels of $\mathrm{A} \beta 42$ and $\mathrm{A} \beta 40$ were reduced in $\mathrm{AD}$ dementia compared with all other diagnostic groups 
* Nakamura, A., et al., High performance plasma amyloid-beta biomarkers for Alzheimer's disease. Nature, 2018. 554(7691): p. 249-254 - This articles demonstrates with high accuracy using a validated IP-MS method to predcit A $\beta$ burden using APP699-711/A $\beta 42$ ratio. 